\title{
Técnica para preparação e conservação de olhos de porco para cirurgia experimental
}

\author{
Technique for the preparation and conservation of pig eyes for experimental surgery
}

\author{
Heryberto da Silva Alvim ${ }^{1}$ \\ Cristiano Menezes Diniz ${ }^{1}$ \\ Patrick Frensel de Moraes Tzelikis ${ }^{1}$ \\ Roberto Martins Gonçalves ${ }^{1}$ \\ João André da Costa Maia ${ }^{2}$
}

Trabalho realizado no Departamento de Oftalmologia do Hospital São Geraldo, UFMG.

${ }^{1}$ Residente de Oftalmologia, Hospital São Geraldo, Universidade Federal de Minas Gerais.

2 Professor Associado de Oftalmologia da Faculdade de Medicina da Universidade Federal de Minas Gerais.

Endereço para correspondência: Av. José Bonifácio 1111, apt. 72 - Campinas (SP) CEP 13093-240 E-mail: alvimhs@yahoo.com

Recebido para publicação em 20.03.2002 Aceito para publicação em 07.10.2002

Nota Editorial: Pela análise deste trabalho e por sua anuência na divulgação desta nota, agradecemos ao Dr. Eduardo Ferrari Marback.

\begin{tabular}{|c|}
\hline RESUMO \\
\hline $\begin{array}{l}\text { Objetivos: Avaliar a eficácia do Dextran } 40 \text { a } 10 \% \text { em promover transparên- } \\
\text { cia corneana em olhos de porco, bem como indicar o melhor método [soro } \\
\text { fisiológico a } 0,9 \% \text { (SF a } 0,9 \% \text { ), Dextran } 40 \text { a } 10 \% \text { ou mistura de ambos em } \\
\text { partes iguais] de conservação para manutenção desta transparência. } \\
\text { Métodos: Vinte olhos de porco, preparados com injeção de Dextran na } \\
\text { câmara anterior e embebidos na mesma solução por } 20 \text { minutos. Quatro } \\
\text { grupos de olhos, cada um com } 5 \text { olhos, conservados nas seguintes } \\
\text { soluções: Grupo } 1 \text { (SF a } 0,9 \%) \text {, Grupo } 2 \text { (SF a } 0,9 \%+\text { Dextran } 40 \text { a } 10 \%) \text {, } \\
\text { Grupo } 3 \text { (Dextran } 40 \text { a } 10 \%) \text {, Grupo } 4 \text { (câmara úmida-grupo controle). Os } \\
\text { olhos foram observados após } 12,24,36,48,56 \text { e } 72 \text { horas e classificados } \\
\text { quanto à transparência da córnea. Resultados: O tratamento da córnea com } \\
\text { Dextran } 40 \text { a } 10 \% \text { preservou a transparência corneana em } 100 \% \text { dos olhos. } \\
\text { A solução de SF a } 0,9 \% \text { e Dextran em partes iguais obteve maior tempo na } \\
\text { manutenção da transparência, com } 60 \% \text { dos olhos viáveis às } 72 \text { horas. } \\
\text { Conclusão: Dextran } 40 \text { a } 10 \% \text { é eficaz para promover a transparência } \\
\text { corneana em olhos de porcopostmortem. A mistura de SF a } 0,9 \% \text { e Dextran } \\
\text { em partes iguais pode ser utilizada para manter viáveis os olhos de porco } \\
\text { preparados por um período de até } 72 \text { horas. }\end{array}$ \\
\hline
\end{tabular}

Descritores: Córnea; Opacidade da córnea; Dextranos; Preservação de órgãos; Animal; Suínos

\section{INTRODUÇÃO}

O olho post mortem passa por uma série de transformações. Neste estado a bomba endotelial entra em falência e então o equilíbrio de fluidos e íons com o humor aquoso fica comprometido resultando na hidratação da córnea em excesso o que contribuirá para sua opacificação ${ }^{(1)}$. A fim de se aproveitar o olho para a cirurgia experimental é necessário uma boa visualização do conteúdo ocular. Assim temos duas alternativas: ou o utilizamos logo após sua retirada, ou o submetemos a técnicas de preparo da córnea para conservar sua transparência por mais tempo. No entanto, nem sempre é possível conseguir olhos frescos assim como utilizá-los imediatamente. Desta forma, faz-se necessário uma técnica para prepará-los conservá-los a fim de mantê-los viáveis o máximo de tempo possível. Na literatura encontramos diferentes técnicas para o preparo utilizando diversos produtos e submetendo a córnea a diferentes tratamentos. É o caso da solução de Swinger-Kornmehl ${ }^{(2)}$, Plasmasteril(3) ${ }^{(3)}$ No entanto não encontramos propostas para conservação e manutenção destes olhos. Este trabalho traz um método para preparação de olhos de porco utilizando Dextran 40 a 10\%, no sentido de avaliar sua eficácia em promover a transparência corneana, 
assim como comparamos diferentes meios de conservação destes olhos já preparados a fim de que possamos utilizá-los por um tempo mais prolongado.

\section{MÉTODOS}

Utilizaram-se 20 olhos de porco post mortem obtidos em um mesmo frigorífico, retirados imediatamente após o abate, ocorrido no dia de início do experimento. Os olhos estavam envolvidos por tecido periorbitário. Após aproximadamente $2 \mathrm{~h}$ da retirada dos olhos iniciou-se a preparação dos mesmos. O tecido que os envolvia foi retirado ao máximo sem traumatizar o globo ocular restando apenas a conjuntiva.

A preparação da superfície corneana procedeu-se da seguinte maneira:

1. Retirada do máximo de epitélio e da membrana de Bowman. Utilização de lâmina de bisturi $n^{0} 15$ (Becton \& Dickson ${ }^{\circledR}$ ) para desepitelização da córnea sob visualização microscópica (aumento de 10x).

2. Repouso por 15 minutos em recipiente contendo Dextran a $10 \%$ (dextran com peso molecular de 40.000 ) misturado em partes iguais com Soro Glicosado a 5\% deixando a superfície corneana virada para baixo em contato direto com a substância.

3. Paracentese da câmara anterior com agulha de calibre $25 \mathrm{X}$ $6 \mathrm{~mm}\left(\right.$ Becton \& Dickson $^{\mathrm{R}}$ ) permitindo a saída de humor aquoso para a injeção de Dextran a $10 \%$ na câmara anterior com a mesma agulha. Em média foi injetado $0.5 \mathrm{ml}$.

Os 20 olhos preparados foram aleatoriamente separados em grupos de 5 e armazenados em recipientes de plástico. Estes foram preenchidos com diferentes soluções formando 4 grupos:

- Grupo 1: Soro Fisiológico (SF) a 0,9\%

- Grupo 2: $20 \mathrm{ml}$ de SF a 0,9\% + 20ml de Dextran 40 a $10 \%$

- Grupo 3: Dextran 40 a 10\%

- Grupo 4: fundo coberto com gaze úmida (câmara úmida) Grupo controle

O volume de solução colocada nos recipientes foi de $40 \mathrm{ml}$, o bastante para cobrir um terço dos olhos, ou seja, não permitindo que o líquido alcançasse o equador, pois assim eles ficariam apoiados lateralmente e não com a córnea voltada para o líquido. Os olhos foram dispostos em contato e circundados com gaze para garantir que permaneceriam com a córnea em contato com o líquido. Reportando ao grupo 4 a câmara úmida consistiu de gaze embebida em SF a $0,9 \%$. Todos os recipientes foram hermeticamente fechados e refrigerados a $6^{\circ} \mathrm{C}$.

Os olhos foram observados após 12, 24, 36, 48, 56 e 72 horas de conservação e avaliados quanto à transparência da córnea pela biomicroscopia (Lâmpada de fenda Nipo Shipon) a luminosidade na intensidade máxima com aumento de 10 vezes e por apenas um pesquisador. A transparência foi assim classificada:

- Transparente: visualização clara da íris e cristalino incluindo as linhas suturais deste ${ }^{(1)}$.

- Moderadamente transparente: visualização dificultada da íris e cristalino, com pouco detalhamento.
- Pouco transparente: visualização de estruturas de câmara anterior sem definição de detalhes (aspecto esbranquiçado).

- Não transparente: a córnea totalmente opaca, impossibilidade de visualização de quaisquer estruturas de câmara anterior.

Foram designadas notas com a finalidade de melhor quantificar o grau de transparência corneana dos diversos grupos facilitando a análise e interpretação dos dados da seguinte forma: nota 15 para córnea transparente, 10 para moderadamente transparente, 5 para pouco transparente e 0 para não transparente.

Foi desenvolvido um banco de dados utilizando o software EPIINFO 6.04 CDC Atlanta.

\section{RESULTADOS}

Quatro grupos foram estudados num total de 20 olhos. O tratamento da córnea com Dextran 40 a $10 \%$ promoveu a transparência corneana em $100 \%$ dos olhos (Fig. 1 e 2). A solução de SF a $0,9 \%$ e dextran em partes iguais (grupo 2) obteve 2 olhos $(40 \%)$ totalmente transparentes às $36 \mathrm{~h}$, e 3 olhos ainda viáveis para cirurgia (notas 10 e 15) às $72 \mathrm{~h}$. No grupo de Dextran puro (grupo 3 ) às $48 \mathrm{~h}$ não havia olhos úteis para cirurgia. No grupo com SF a $0,9 \%$ (grupo 1) não havia olhos com nota 15 ao fim de $24 \mathrm{~h}$. O grupo 3 não tinha mais olhos com nota $15 \mathrm{em} 12 \mathrm{~h}$, e às $48 \mathrm{~h}$ seus olhos já não eram mais viáveis. Os olhos em câmara úmidos (grupo controle, n. 4) tiveram um comportamento semelhante ao grupo 2 porém após $24 \mathrm{~h}$ não havia mais olhos com transparência nota $15 \mathrm{e}$ às $72 \mathrm{~h}$ havia apenas 2 olhos com transparência útil para cirurgia (notas $10 \mathrm{e}$ 15) (Gráficos 1 e 2).

\section{DISCUSSÃO}

Os olhos de porco, escolhidos pela facilidade de obtenção, possuíam tecido orbitário o que promoveu uma proteção maior

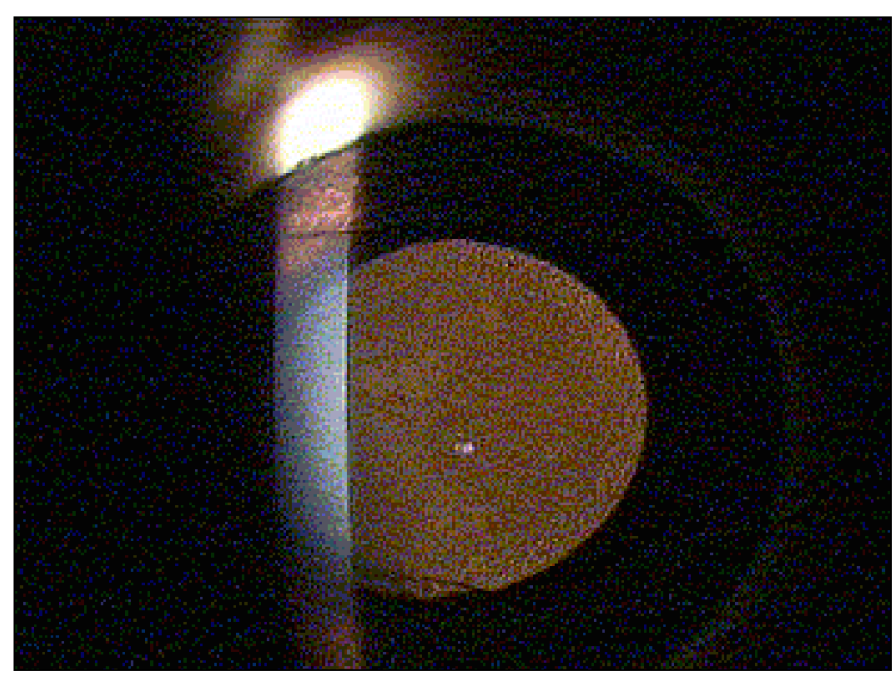

Figura 1 - Antes da preparação 


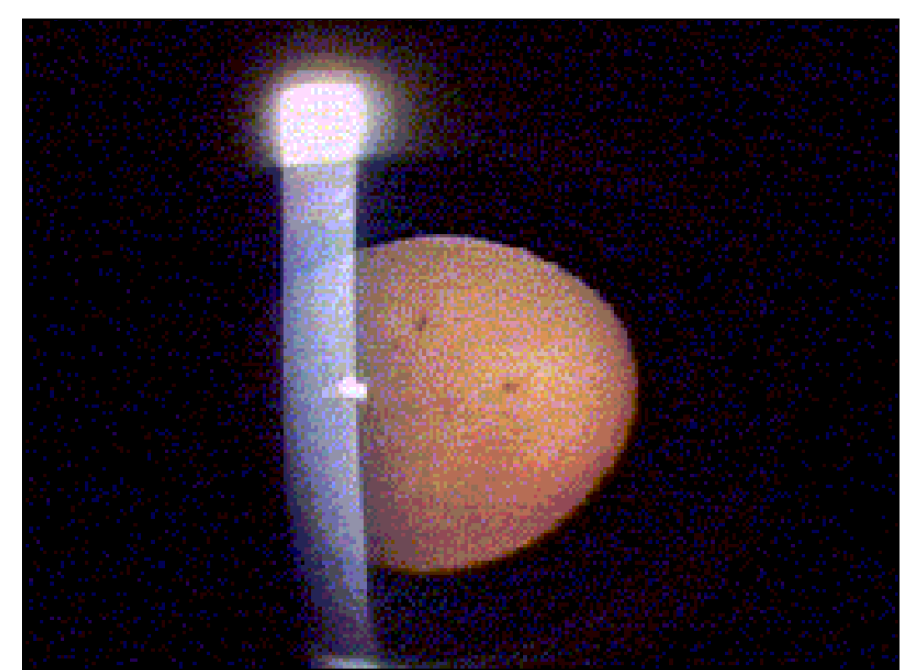

Figura 2 - Após a preparação

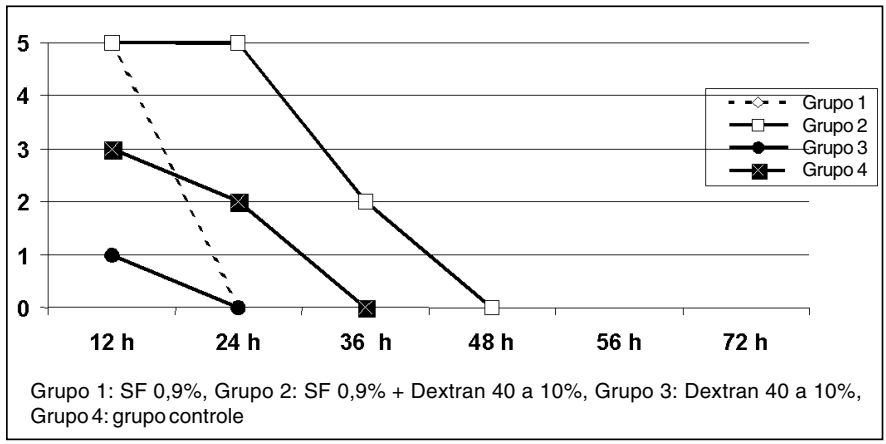

Gráfico 1 - Número de olhos que mantiveram transparência nota 15 ao longo do tempo

ao globo ocular, evitando perfuração ou abrasão na superfície corneana na sua retirada. A desepitelização sob microscopia garantiu uma limpeza mais completa do epitélio. Swinger desepitelizou a córnea e a embebeu por 20 minutos em solução SK( Swinger-Kornmehl). Esta é uma solução de dextran a 30\% em solução salina normal com $0,05 \%$ de metilparaben e $0,005 \%$ propylparaben. A solução Plasmasterilâ, que contém amido hidroxietílico $60 \mathrm{~g}$, foi avaliada em outro estudo para desidratação corneana com resultados satisfatórios ${ }^{(3)}$. Sendo assim, antes da conservação, os olhos foram embebidos por 20 minutos no Dextran. Swinger et al, em 1995 e, posteriormente, Auffarth et al, em 1996, injetaram a solução SK na câmara anterior para preservar a transparência corneana ${ }^{(2,4)}$. A finalidade da colocação de soluções hipertônicas na câmara anterior é mantê-la hiperosmótica em relação à córnea reduzindo a sua hidratação que naturalmente ocorreria por se tratar de olhos post mortem $^{(4)}$. Utilizou-se o Dextran 40 a $10 \%, 500 \mathrm{ml}$, para o preparo dos olhos, pois a solução SK não foi encontrada.

Com os métodos utilizados, não forma encontrados dados sobre a conservação de olhos preparados para cirurgia experimental. Na literatura encontramos que a solução SK é útil para olhos humanos até o $4^{0}$ dia post mortem $^{(4)}$. Outros estudos

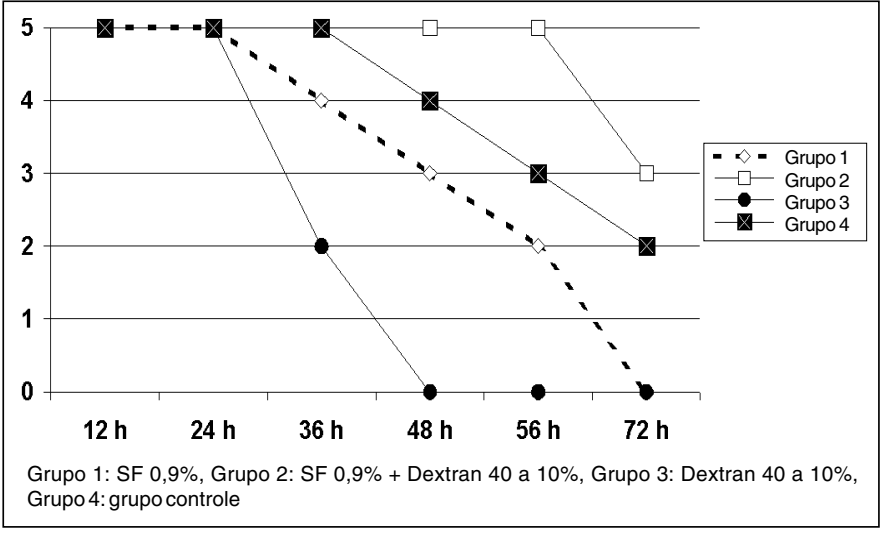

Gráfico 2 - Número de olhos que mantiveram transparência viável para cirurgia (nota 15 e 10) ao longo do tempo

afirmam que o ideal é manipular olhos de porco até $24 \mathrm{~h}$ post mortem devido à turvação corneana que se inicia a partir deste período $^{(5)}$. No entanto não discutem como conservar estes olhos.

A substância conservante deveria permitir menos trocas possíveis com a córnea evitando uma hiperidratação ou desidratação excessiva, onde ambas situações reduziriam a transparência corneana. Assim foram testados SF a 0,9\%, Dextran 40 a $10 \%$ e a mistura de ambos em partes iguais, além de um grupo controle.

Considerou-se que apenas os graus transparente e moderadamente transparente permitem uma boa visibilidade para realização de cirurgia experimental.

A respeito dos métodos de conservação, o mais eficiente foi a mistura em partes iguais de Dextran 40 a $10 \%$ e SF a $0,9 \%$. Esta propiciou um maior número de olhos com notas 15 e 10 de transparência por um tempo mais prolongado (Gráficos 1 e 2). A solução com piores resultados foi a de Dextran puro onde, ao fim de 24 horas, alguns olhos já estavam inviáveis para cirurgia experimental e, após 48 horas, todos os olhos tiveram nota 5 de transparência da córnea. O grupo controle obteve resultados semelhantes ao da solução SF a 0,9\% e Dextran 40 a $10 \%$. A conservação com $\mathrm{SF}$ a $0,9 \%$ mostrou resultados intermediários entre as soluções anteriores.

A diferença de transparência foi atribuída às variações de concentração de dextran nas soluções criadas. Adicionando SF a $0,9 \%$ a esta solução, reduzimos seu poder osmótico levando a uma menor desidratação da córnea o que lentifica o processo de perda de transparência post mortem. A utilização do SF a 0,9\% isoladamente produz efeito contrário, hidratação do estroma corneano que, se contínuo, como no processo de conservação, leva a hiperidratação prejudicando a transparência.

Alguns estudos avaliaram o efeito do dextran no endotélio corneano ao ser adicionado em meio de conservação para córnea $^{(6-7)}$. Após 1 semana nesta solução contendo Dextran é possível observar múltiplas necroses endoteliais em metade das córneas conservadas. Microscopicamente identifica-se grânulos e vacúolos intracitoplasmáticos, depósitos na su- 
perfície endotelial e alargamento dos espaços intercelulares, além de polimorfismo diminuição da contagem endotelial.

No entanto não são apenas as alterações endoteliais as responsáveis pela perda de transparência e apesar da toxicidade endotelial vários autores estudaram os benefícios do Dextran na córnea. A redução na composição de proteoglicans (PGs) estromais já foi anteriormente demonstrada nos casos de edema corneano, cicatrização e doenças. É sabido que também ocorre perda dos PGs durante a preservação das córneas, no entanto é menor quando se adiciona Dextran na solução de preservação ${ }^{(8)}$. Este grupo observou que todas as córneas estavam hidratadas acima do normal porém o grupo com dextran apresentava-se em menor intensidade, tornandoas de melhor qualidade.

Barrett et al utilizou agentes osmóticos na tentativa de restaurar a espessura de córneas "doadoras" de porco a seu estado in $v i v o^{(9)}$. Notou que a conservação destas córneas em solução de preservação McCarey-Kaufman (MK, contém dextran e sulfato de condroitina) resultava em aumento da espessura corneana em torno de $50 \%$. Seu estudo provou que o dextran a $15 \%$ sozinho ou a $10 \%$ associado a sulfato de condroitina reduziu a espessura corneana ao seu estado in vivo. Outro estudo conduzido por Lass et al também avaliou a eficácia e segurança do Dextran quando adicionado à solução de preservação corneana ${ }^{(10)}$. A adição do dextran resultou em significativa redução da espessura corneana no intra-operatório sem efeitos adversos na síntese de DNA in vitro e na sobrevida endotelial in vivo.

Duffey et al realizaram um interessante estudo no qual demonstraram que o Dextran foi eficaz em desidratar a córnea quando injetado na câmara anterior permitindo que a córnea permanecesse por até 1 hora com uma espessura de 500 a 600 microns, o que permitia dinâmica corneana consistente para cirurgia refrativa experimental ${ }^{(11)}$.

\section{CONCLUSÃO}

O uso do Dextran 40 a 10\% foi útil para promover a imediata transparência corneana através da sua injeção na câmara anterior e exposição da superfície desepitelizada a esta substância. No entanto utilizá-lo puro para a conservação não constitui uma boa opção. A conservação do olho em solução de partes iguais de SF a $0,9 \%$ e Dextran 40 a 10\% (Grupo 2) foi superior aos demais meios, sendo uma medida eficaz nos casos em que os olhos não forem utilizados em 24 horas uma vez que esta mistura permite que haja olhos viáveis para cirurgia experimental em até 72 horas. Isto poupa a equipe do trabalho de coleta permitindo um melhor aproveitamento do material já obtido.

\section{AGRADECIMENTO}

Ao Dr. Paulo de Tarso Silva Alvim pelo incentivo a criação deste trabalho, fornecendo materiais que incitaram a idéia inicial do experimento.

\section{ABSTRACT}

Purposes: To evaluate Dextran 40 10\% efficacy to maintain pig eye corneal transparency in order to indicate the best method $(0.9 \%$ physiological saline, Dextran $4010 \%$ and the mixture of both at equal parts) of conservation to maintain the transparency. Methods: Twenty pig eyes, all of them prepared with Dextran injection in the anterior chamber and soaked in this solution for 20 minutes. Four groups, each group with five eyes and kept at $6^{\circ} \mathrm{C}$ in the following solutions: Group $1(0.9 \%$ physiological saline), Group 2 (Dextran $4010 \%+0.9 \%$ physiological saline at equal parts), Group 3 (Dextran 40 10\%), Group 4 (humid chamber - control group). The eyes were observed after 12, 24, 48, 56, 72 hours and then classified according to their transparency. Results: Corneal transparency occurred in $100 \%$ of the eyes with Dextran solution. $0.9 \%$ physiological saline and Dextran at equal parts achieved a longer period of transparency. Conclusions: Dextran $4010 \%$ is an efficient solution to promote corneal transparency in post mortem pig eyes. The mixture of equal parts $0.9 \%$ physiological saline and Dextran can be used to keep feasible the prepared eyes for a period as long as 72 hours.

Keywords: Cornea; Corneal opacity; Dextrans; Organ preservation; Animal; Swine

\section{REFERÊNCIAS}

1. Oréfice F, Boratto LM, eds. Biomicroscopia e gonioscopia. Rio de Janeiro: Cultura Médica; 1989. p. 72-4.

2. Swinger CA, Kornmehl EW. Dehydration of post-mortem eyes for practice and experimental surgery. Ophthalmic Surg 1985;16:182-3.

3. Silva HMB, Bertotti GSA, Freitas JAH, Piccinin MRM, Camargo MSF. Viabilizando a cirurgia experimental de catarata em olhos humanos enucleados. Rev Bras Oftalmol 2000;59:404-8.

4. Auffarth GU, Wesendahl TA, Solomon KD, Brown SJ, Apple DJ. A modified preparation technique for closed-system ocular surgery of human eyes obtained postmortem: an improved research and teaching tool. Ophthalmology 1996;103:977-82.

5. van Vreeswijk H, Pameyer JH. Inducing cataract in postmortem pig eyes for cataract surgery training purposes. J Cataract Refract Surg 1998;24:17-8.

6. Lin CP, Böhnke M, Draeger J. Effect of Dextran on predamaged corneal endothelium: an organ culture study. Ophthalmic Res 1992;24:125-8.

7. Abramo F, Böhnke M, Draeger J. Morphology of the corneal endothelium following preservation in dextran or chondroitin sulfate containing culture media. Fortschr Ophthalmol 1990;87:234-6.

8. Slack JW, Kangas TA, Edelhauser HF, Geroski DH, McDermott ML. Comparison of corneal preservation media for corneal hydration and stromal proteoglycan loss. Cornea 1992;11:204-10.

9. Barrett GD, Degebrodt AM, Walker LN. Improved storage methods for epikeratoplasty. Refract Corneal Surg 1990;6:418-23.

10. Lass JH, Reinhart WJ, Skelnik DL, Bruner WE, Shockley RP, Park JY, Hom DL, Lindstrom RL. An in vitro and clinical comparison of corneal storage with chondroitin sulfate corneal storage medium with and without dextran. Ophthalmology 1990;97:96-103.

11. Duffey RJ, Tchah H, Lindstrom RL. Human cadaver corneal thinning for experimental refractive surgery. Refract Corneal Surg 1989;5:41-2. 